\title{
LAS REVISTAS EDUCATIVAS PERUANAS Y SU CONTRIBUCIÓN AL ANÁLISIS DE LA PROBLEMÁTICA MAGISTERIAL
}

\section{Peruvian educational journals and their contribution to the analysis of the educational problem}

\author{
Gerber Sergio Pérez Postigo \\ Universidad Nacional de San Agustín de Arequipa - Perú \\ Correo: gperezpo@unsa.edu.pe \\ Jorge Luis YANGALI VARgas \\ Universidad Nacional del Centro del Perú \\ Correo:jyangali@uncp.edu.pe \\ Osbaldo Washington Turpo Gebera \\ Universidad Nacional de San Agustín de Arequipa - Perú \\ Correo: oturpo@unsa.edu,pe
}

Recibido: 13 de noviembre de 2020

Envío a informantes: 20 de noviembre de 2020

Aceptación definitiva: is de enero de 202I

RESUMEN: En el presente artículo damos a conocer las características de las revistas publicadas entre siglos, vale decir, las que comenzaron en la última década del siglo xx y las que emergieron en estas dos primeras décadas del nuevo siglo xxI. Las revistas educativas peruanas, otrora impresas, han migrado significativamente hacia la virtualidad; las que no lo hicieron han reducido la visibilidad de los artículos académicos que en ellas se publican. El mayor número de estas revistas están vinculadas a las universidades como agencias editoriales; no obstante, también se tienen revistas asociadas a organizaciones sociales, al Gobierno y al sindicato docente. Estas últimas conservan su función divulgativa y generadora de debate sobre las políticas públicas; en tanto que las otras se muestran como revistas que comunican los resultados de las investigaciones en el campo educativo.

Palabras clave: revistas académicas; formación docente; universidades; indexación; acceso a la información. 
АвsтRAст: In this article, we present the characteristics of the journals published between the last decade of the 2oth century and those that emerged in the first two decades of the 2Ist century. Peruvian educational magazines, once printed, have migrated significantly towards the virtual mode. Those that did not have reduced the visibility of the academic articles that were published in them. Most of these journals are linked to universities as editorial agencies; however, there are also magazines associated with NGOs, the government, and the teachers' union. This last one maintains two functions, informative and generator of debate on public policies; while the others are shown as journals that communicate the results of research in the educational field.

KEY WORDs: scientific journals; teacher training; university colleges; indexing; access to information.

\section{Introducción}

$\mathrm{L}$

A PROBLEMÁtica EDITORIAL en educación viene afrontando, en los últimos años, exigencias significativas y que están siendo asumidas según la naturaleza de las instituciones que patrocinan dichas publicaciones. En este tiempo de pandemia, la problemática se ha agudizado, toda vez que las revistas que tenían como único formato el impreso han visto suspendidos sus mecanismos de distribución y, en algunos casos, de venta.

En el Perú, el acceso a los contenidos educativos de dichas revistas no se ha visto afectado en este periodo de confinamiento por la pandemia, toda vez que en los últimos años las entidades editoriales han migrado al formato virtual. Cabe precisar que el modelo de publicación en acceso abierto en Latinoamérica se sostiene por el financiamiento de las casas editoras. No obstante, en razón a los costos que exige la publicación, pocas revistas han establecido tasas por publicación que son asumidas por los autores.

En ese contexto de renovación editorial, el sistema de formación magisterial peruano continúa por el derrotero legado por la Reforma Educativa de 1969, con el ideario de masificar la educación en todo el territorio peruano, impulsando la incorporación de docentes en todos los niveles educativos, recurriendo para ello al nombramiento interino de profesores, quienes en un gran número no contaban con formación docente alguna. Por lo mismo, se diseñaron programas de capacitación docente, jugando, en esta tarea, un valioso rol las revistas tanto de las universidades públicas como de los otros sectores asociados al magisterio, entre los que se cuentan el sindicato, las organizaciones no gubernamentales (ONG) y el mismo Gobierno.

Situados en este marco contextual, se nos plantea como objetivo dar a conocer los rasgos de las revistas educativas peruanas y su contribución, tanto en la visibilidad de la producción académica local, como en el abordaje de la problemática magisterial.

\section{Métodos}

Para sistematizar el presente artículo monográfico, primeramente, hemos realizado búsquedas hemerográficas en los archivos institucionales de las revistas impresas como en los portales web de las editoriales encargadas de su publicación. Ubicados 
LAS REVISTAS EDUCATIVAS PERUANAS Y SU CONTRIBUCIÓN AL ANÁLISIS DE LA PROBLEMÁTICA MAGISTERIAL

G. S. PÉREZ POSTIGO, J. L. YANGALI VARGAS Y O. W. TURPO GEBERA

los medios impresos y digitales, los dividimos entre aquellas revistas que no han sido indizadas y las que sí se encuentran en alguna base de datos. Entre las primeras contamos con revistas vinculadas al sindicato docente y entre las indizadas se cuentan las revistas afiliadas a las universidades u otra entidad académica.

\section{Resultados}

\section{I. Palabra del sindicato peruano}

Palabra de Maestro es una revista editada por la Derrama Magisterial, institución de Seguridad Social privada, perteneciente a los maestros del Perú que trabajan en las instituciones educativas del Estado. Fue creada el ro de diciembre de 1965 , a solicitud de los gremios de maestros para contar con un Fondo de Retiro que incremente los beneficios previsionales que otorgaba el sistema estatal. Comenzó a funcionar desde el I de marzo de 1966.

Desde 1990 la Derrama Magisterial inicia la publicación de la revista Palabra de Maestro, con el propósito de poner en relieve las preocupaciones profesionales de los maestros del Perú sobre la mejora continua y actualización magisterial; desde 1990 hasta el 2017 Palabra de Maestro publicó 66 números y desde noviembre del 20I7, que cambia el nombre de la revista a $A D$ Maestro, inicia nuevas publicaciones y que hasta la actualidad cuenta con la edición de iz números.

Entre sus objetivos resalta la publicación de números trimestrales y abiertos a recibir colaboraciones no solo de los expertos en educación, sino también de diversas participaciones, quienes hacen llegar sus artículos, cartas y/o comentarios, desde distintos puntos del país, acerca de sus principales áreas de trabajo, experiencias y preocupaciones relacionadas con el ejercicio de la profesión docente. Entre los principales temas de debate en torno a la educación en el Perú se puede señalar los siguientes.

\subsection{Ley de Reforma Magisterial ¿Afrontando el problema de los docentes?}

En Palabra de Maestro, 59 (2013), se analiza la Ley de Reforma Magisterial, con la participación de especialistas, juristas y dirigentes del Sindicato Único de Trabajadores de Educación del Perú (suTeP). Entre los hallazgos resalta el derecho de los miembros del magisterio a percibir una bonificación mensual, por preparación de clases y evaluación, equivalente al 30\% de su remuneración total. Este beneficio ha sido considerado por sentencias del Tribunal Constitucional. «Si en la práctica lo que establece la Ley de Reforma Magisterial implicase un recorte salarial, es indudable que su aplicación significaría un contrafuero inconstitucional. Las conquistas laborales del magisterio son irrevocables», manifestó Valle Riestra.

El profesor René Ramírez Puerta, secretario general del SUTEP, señala que la Ley de Reforma Magisterial solo hace parches a la Ley de Carrera Pública Magisterial, dentro de sus argumentos señala:

¿Por qué se oponen a la Ley de Reforma Magisterial? Porque además de haber sido impuesta, de no haber sido consensuada con los maestros, solamente hace algunos par- 
ches a la Ley de Carrera Magisterial 29062. Con esta norma los neoliberales que gobiernan el país buscan tres objetivos: ahorro fiscal, para seguir reduciendo gastos en el sector y seguir pagando la deuda externa; profundizar la desregulación laboral de los docentes al pretender convertirnos en profesionales con títulos provisionales; y continuar agudizando la pobreza extrema en la que viven miles de educadores, a quienes se nos condena a seguir con sueldos muy bajos y, por necesidad ser contratados en colegios particulares donde la explotación al docente ya no tiene límites.

$¿$ Considera que hay algunos aspectos positivos a la Ley? El espíritu de esta Ley no tiene nada de positivo. Si bien es cierto en un primer momento traerá para los maestros de 30 horas unos cuantos soles más, lo que no será para los de 24 horas, anula derechos adquiridos y, sobre todo humilla al magisterio al rebajarlos de nivel, sin tener en cuenta que este fue alcanzado en cumplimiento de una Ley, la del Profesorado.

La nueva Ley, ¿̇recoge algo de la propuesta de Nueva Ley del Profesorado, del SUTEP? Solo la evaluación del desempeño sobre el trabajo del profesor en el aula, a cargo de una Comisión en cada Institución Educativa, aunque marginando la representación gremial, esta Ley no recoge nada de lo que el SUTEP ha propuesto. El debate en el Congreso ha sido solo en torno a la propuesta del gobierno. Los congresistas no tomaron en cuenta nuestra propuesta.

Julio Mendoza García, miembro de la Comisión de Elaboración de la Nueva Ley del Profesorado del SUTEP, plantea que las políticas magisteriales deben ser Políticas de Estado. Deben trascender al Gobierno, expresar un consenso social y tener la legitimidad política.

\section{3. ¿Cambios en la docencia? Retos y tensiones}

En Palabra de Maestro, 6I (20I4a) se hace un análisis sobre los nuevos escenarios y demandas para la formación docente y en ella se sostiene que tenemos la plena convicción de que un profesor de matemática o de arte, de escuela rural o urbana, con alumnos de habla castellana o de lenguas originarias debe tener claro que su actuar educativo está ligado al desarrollo del país y tener la suficiente convicción política para optar por un determinado tipo de desarrollo. Un profesor debe, sobre todo, tener un conocimiento de la sociedad en la que desarrolla su labor y desde este conocimiento estar en capacidad de emprender el desarrollo integral en lo físico, social, mental, afectivo y espiritual de los niños, jóvenes y adultos a su cargo.

Luisa Pinto (20I4a) señala que el reto que plantea el desarrollo sostenible es hoy mayor que nunca. Los avances tecnológicos, legislaciones y los marcos políticos no bastan. Necesitamos cambios en las mentalidades, valores y estilos de vida, y fortalecer la capacidad transformadora de las personas.

El rol del maestro de hoy es desafiante: motivar y entretener supone entusiasmo y convicción sobre la importancia de su quehacer. La educación es un diálogo entre generaciones. Es una tarea central en la reproducción de la sociedad.

Sigfredo Chiroque Chunga, investigador del Instituto de Pedagogía Popular, señala que en el Perú las políticas magisteriales de corte neoliberal recién se hicieron explícitas con mayor claridad en el año 2007 con la Ley n. ${ }^{\circ} 29062$ de Carrera Pública Magisterial, impuesta por el Gobierno aprista. La Ley de Reforma Magisterial $n .{ }^{\circ}$ 29944 promulgada por el Gobierno de Ollanta Humala, en noviembre del 20I2, ace- 
LAS REVISTAS EDUCATIVAS PERUANAS Y SU CONTRIBUCIÓN

AL ANÁLISIS DE LA PROBLEMÁTICA MAGISTERIAL

G. S. PÉREZ POSTIGO, J. L. YANGALI VARGAS Y O. W. TURPO GEBERA

leraría la aplicación de las principales medidas de la LCPM. En realidad, ninguna de las dos leyes estableció políticas de pleno desarrollo docente, sino que se focalizó en regular los desempeños docentes y la Carrera Pública Magisterial.

Manuel Bello, profesor principal de la Facultad de Educación de la Universidad Peruana Cayetano Heredia, sobre la Formación Docente se pregunta: ¿A dónde queremos que llegue la educación en las dos próximas décadas? ¿Cómo podremos saber si estamos avanzando para lograr la visión educativa que nos hemos propuesto como sociedad? ¿Son los docentes los responsables de los pésimos resultados en la evaluación PISA? ¿Qué hacemos frente a la influencia hegemónica de las fuerzas del mercado, que han convertido a la educación en una mercancía y no en un derecho social, creando la llamada «segregación (o apartheid) escolar»?

Sobre el desempeño docente y aspectos sistémicos de la Educación, plantea que lo que hacen y dejan de hacer los docentes en la institución educativa es muy importante, pero los resultados del aprendizaje de los estudiantes no dependen solo del desempeño de los profesores. La familia y la comunidad cumplen un rol importante, los medios de comunicación masiva también tienen un papel. En la propia escuela, los aprendizajes también dependen de la disponibilidad de recursos y materiales educativos, tecnología, textos, infraestructura adecuada, además de buen clima y organización institucional.

En Palabra de Maestro, 63 (20I4b) se publican diferentes artículos que tratan sobre diferentes aspectos de la educación, entre algunos de ellos podemos destacar los siguientes:

\subsection{Estos virus nunca se han ido}

Se publica una entrevista al psicoanalista Jorge Bruce sobre el cambio de conducta y comportamientos de la sociedad peruana en relación con los resultados electorales del 5 de octubre, donde muchos ciudadanos optaron por alternativas vinculadas a la corrupción. ¿Qué pasó? ¿Nos estamos viendo tolerantes a este terrible flagelo como lamentablemente lo somos al racismo y la discriminación? ¿Cómo entender estos fenómenos sociales y cuál es nuestro rol como educadores?

Frente a estas interrogantes señala que esto no es un fenómeno nuevo; es posible, sin embargo, que se haya consolidado. Por ejemplo, en la ciudad de Lima, a muchos alcaldes electos se les ha visto con grilletes en la mano, algunos elegidos por quinta vez con sospechas de corrupción y entonces uno se pregunta: ¿Qué pasa? ¿La gente es absolutamente impermeable a la corrupción?

Lo que está claro es que muchas personas optan por este tipo de representantes porque en sus sumas y restas, estos los van a beneficiar en lo inmediato. Y, además hay esta creencia extendida de que todos roban, por tanto, a partir de ese rasero, quién es el que me puede proporcionar más servicios, considerando que el Estado en general es muy ineficiente en los servicios que presta. Entonces, esta es una de las cosas que contribuye a que las personas hagan la vista gorda ante casos sonados.

El resentimiento siempre estará presente en todas las sociedades, porque a mayor desigualdad es evidente que habrá mayor resentimiento. Es un sentimiento que es inevitable que exista. Y la mejor manera de combatirlo es justamente combatiendo la desigualdad. Si vivimos en los abismos de la desigualdad, yo no sé cómo podríamos 
evitar esos efectos negativos, y no solo el resentimiento, sino también la envidia, la desconfianza, el miedo.

Las personas que más se integran al modelo se vuelven más individualistas, porque eso es finalmente lo que el modelo propugna. Si enfrentamos todo esto, estamos dejando a nuestros descendientes un país que va a ser más difícil de manejar, y esta sí puede ser la versión más catastrófica, expresa el psicoanalista.

En un modelo hegemónico como el actual, donde lo que prima es el sistema darwiniano de la ley del más fuerte, y donde el éxito es medido con la cantidad de dinero, propiedades, influencia y poder, se favorece el posicionamiento de figuras que tienen características vinculadas a la psicopatía, es decir, personas que son implacables y que son capaces de hacer lo que sea porque el fin justifica los medios.

\subsubsection{Sistema educativo peruano y visión del currículo}

Óscar Huaranga Ross (20I4b), asesor pedagógico de INFODEM, señala que el currículo es, a la vez, análisis y propuesta de sociedad y del hombre que se proyecta en el horizonte, buscando comprender por qué está cambiando nuestra manera de hacer las cosas: trabajar, divertirnos, relacionarnos, aprender; y cómo, de manera sutil, también varía nuestra forma de pensar. El currículo se concibe como un proyecto de vida y los valores que se deben privilegiar en la comunidad educativa. Debe tener una orientación educativa acorde con las demandas de la sociedad donde se desenvuelve el alumno, de allí el interés por conocer la intencionalidad social del currículo. Hay aspectos que no deben sobreentenderse y que deben ponerse en práctica a través del quehacer pedagógico del docente.

Antecedentes históricos del currículo. A lo largo de la historia de la educación en el país, han existido diversas maneras de expresar los propósitos educativos, en dependencia de los especialistas o diseñadores curriculares y la ideología curricular dominante y hegemónica. Desde la década de los años 6o, del siglo pasado, predominó el modelo curricular de la Tecnología Educativa, el cual se sustentaba en varios enfoques: por objetivos, experiencias de aprendizaje, contenidos temáticos y por habilidades y destrezas.

La globalización nos pone en condiciones de competitividad que desafían los aprendizajes y la enseñanza, lo que nos lleva a reflexionar sobre la razón de ser de un sistema educativo y curricular, bajo las ideas de derecho a la Educación o convertirla en mercancía.

Existen dos visiones sobre el desarrollo humano y el aprendizaje: una con enfoque individualista y énfasis en lo cognitivo (inteligencia) para el mercado; y otra, desde una personalizada y holística (integral) de enfoque humanista para la ciudadanía.

\subsubsection{Corrientes curriculares en América Latina}

El profesor Carlos Rojas Galarza (20I4b), de la Universidad Nacional Enrique Guzmán y Valle, señala que el tema del currículo siempre suscita debate, la atención investigativa a nivel intensivo y extensivo de las diferentes direcciones conceptuales y experimentales. Por tanto, se han presentado un conjunto de planteamientos inno- 
LAS REVISTAS EDUCATIVAS PERUANAS Y SU CONTRIBUCIÓN AL ANÁLISIS DE LA PROBLEMÁTICA MAGISTERIAL

G. S. PÉREZ POSTIGO, J. L. YANGALI VARGAS Y O. W. TURPO GEBERA

vadores convergentes y divergentes en el mundo, particularmente en América Latina, los cuales son revisados en el siguiente artículo.

Como lo señalan connotados científicos sociales, Latinoamérica es una región de inmensas diferencias económicas, sociales, culturales y geográficas, aun cuando en términos genéricos es considerada como una sociedad heterogénea emergente tardía por la condición histórica de ser dependiente de potencias como Estados Unidos de Norteamérica y organismos financieros internacionales como el FMI, el BM, el BID, etc.

En el Perú, en los últimos 25 años de instauración del modelo neoliberal en la región, se ha producido una variedad de políticas educativas que van desde las más pegadas a los mandatos del Banco Mundial y el Banco Interamericano de Desarrollo, como ocurrió con el Gobierno de Alberto Fujimori, principalmente. Al respecto, IPEBA (Instituto Peruano de Evaluación, Acreditación y Certificación de la Calidad de la Educación Básica) ha descrito la vinculación de los organismos internacionales financieros y su intromisión en la educación peruana con las siguientes afirmaciones: Los primeros diez años de estudio (de IPEBA) corresponde al Gobierno de Alberto Fujimori (1990-200I). Hubo un cambio político en la escena nacional, e imperaba una política neoliberal. Al inicio de esta administración, la economía peruana se insertó en la economía mundial y se inició un proceso de reforma del Estado.

En resumen, a la política económica de mercado libre, correspondió la política educativa pragmática, revelada en un currículo constructivista-cognitivo, sin fundamentos filosóficos, epistemológicos, sociológicos, psicopedagógicos y con anulación progresiva de asignaturas como filosofía y psicología en el nivel secundario. Así mismo se restringieron al mínimo los contenidos de las asignaturas de historia, geografía y economía.

\subsubsection{Formación continua y evaluación docente}

En Palabra de Maestro 66 (2016) se publican los planteamientos señalados en una mesa redonda organizada por la Derrama Magisterial sobre la Formación Continua y Evaluación docente, con la participación de Luis rodríguez de los Ríos, catedrático de posgrado de la Universidad Enrique Guzmán y Valle; Magaly Robalino, representante de Unesco en Perú, y Marco Raúl Mejía, experto colombiano.

Según Rodríguez de los Ríos, no existe acuerdo ni claridad en la forma de denominar y conceptualizar la formación continua de profesores. Sí hay una variedad de propuestas como formación durante la vida laboral, en servicio, desarrollo profesional, formación permanente, actualización, capacitación, perfeccionamiento, superación, etc. «En el Perú se busca promover las competencias del Marco del Buen Desempeño Docente», señaló. Para él, la formación continua es el proceso institucionalizado en varias modalidades, desde la formación inicial y el posgrado; mientras que la formación permanente, según Paz (2005), es un proceso inherente a la práctica (y teoría) cotidiano de los profesionales de la educación:

Que promueve la autoformación, no tiene como exigencia un programa prefijado y depende más de las motivaciones de los docentes que de lo externo. Lamentablemente los programas de formación magisterial no consideran que el servicio profesional docen- 
te deba sostenerse en la formación continua de sus actores, sino que orienta a la acumulación indiscriminada de puntajes, diplomas y horas de formación.

Tampoco contempla el nexo determinante entre Formación Magisterial, capacidades en los docentes, desempeños docentes y logros de aprendizaje en los estudiantes, «siendo necesario señalar el derrotero y relatividad de cada uno de estos factores, para poder trazar políticas educativas, sin menoscabar el peso de cada uno de ellos».

El experto rescató las recomendaciones del Consejo Nacional de Educación al afirmar que el Ministerio de Educación no tiene un concepto claro de lo que es formación docente en servicio, por lo que se debe participar consensuando una propuesta.

Así mismo señaló que urge identificar las experiencias exitosas de capacitación desarrolladas descentralizadamente, difundirlas y replicar las lecciones aprendidas, recomendó:

Elaborar políticas nacionales de capacitación y especialización docente que estén a cargo de los formadores con experiencia de las regiones, UGEL, ONG, centros Amauta, universidades, pedagógicos e incluirlas dentro de las políticas articuladas del Proyecto Educativo Nacional y proyectos educativos regionales, entre otras.

La formación continua se ampara en el trabajo colaborativo, la indagación y experimentación y la reflexión crítica sobre la propia cultura y práctica de la escuela, y se orienta hacia las comunidades, más que hacia los docentes de forma individual. El desarrollo profesional supone articular la potenciación de las nuevas competencias a adquirir junto al proceso de cambio, mejora de la institución, mejoramiento de la calidad educativa y logro de aprendizajes significativos en los estudiantes. Asimismo, se insiste:

Queremos docentes comprometidos con la inclusión educativa, preparados para enseñar en la diversidad. Es momento de repensar su rol desligándolo de ser un ejecutor mecánico hacia un nuevo profesor más activo, capaz de tomar decisiones, transformador, innovador y más creativo.

Sobre la evaluación docente dijo que tiene connotación política: «¿Evaluarlos para despedirlos? ¿Así se resolverán los problemas de la educación? Creemos que no. Por eso es urgente lograr consenso y la participación docente en la construcción de la propuesta evaluativa, para darle mayor legitimidad».

Recomendó establecer claramente el modelo que promueve el sistema educativo vigente para determinar qué es lo que se espera del docente. Esto es fundamental no solo para la evaluación, sino para el diagnóstico real de la formación de los docentes a nivel inicial, continuo y permanente.

A su turno, Magaly Robalino señaló que América Latina y el Caribe están reconociendo el lugar estratégico de los docentes y directores en el aseguramiento de una educación de calidad. Podríamos decir que estamos en una etapa de reencuentro con los docentes, como afirma Novoa (2009).

Hay una tendencia hacia la formación docente en el nivel universitario, y se están dando leyes que incluyen el subsistema de formación inicial gubernamental bajo la dirección de los ministerios del sector y norman el funcionamiento, la evaluación y la acreditación... Sin embargo, vemos predominio de carreras de formación docente 
LAS REVISTAS EDUCATIVAS PERUANAS Y SU CONTRIBUCIÓN AL ANÁLISIS DE LA PROBLEMÁTICA MAGISTERIAL

G. S. PÉREZ POSTIGO, J. L. YANGALI VARGAS Y O. W. TURPO GEBERA

para humanidades, y menor proporción para ciencia y tecnología. Alrededor del $75 \%$ del magisterio posee certificación para la docencia en primaria, y aproximadamente el $65 \%$ en secundaria (Unesco/UIs, 2009).

Se observa como aspecto crítico que «La formación docente está alejada de la profesión docente y no responde a un proyecto de desarrollo», que la formación inicial está relegada en los proyectos nacionales, por el énfasis de los programas de formación en servicio y cuestionamiento a las instituciones formadoras, indicó la funcionaria de Unesco.

Asimismo «vemos desvinculación de la formación docente con las tecnologías de información y comunicación, y la insistencia de valorizar la profesión docente sin una mejora salarial».

Frente a ello, dijo que es necesario una visión transformadora de las instituciones formadoras. Deben convertirse en uno de los motores de transformación de la educación (Aguerrondo y Pogré, 2000). Pasar del trabajo solitario a la articulación con niveles clave del sistema educativo: currículo, formación continua, normativa, etc. Ser gestores de innovación pedagógica en investigación, información web, producción y circulación de conocimiento.

Es necesario darle a la formación docente su lugar en el proyecto educativo nacional y en un proyecto de país; por tanto, en el sistema educativo.

Definir el tipo de docentes que el país requiere. Enfoques, estrategias, contenidos, metodologías. Es fundamental tener claro qué es un buen profesor y qué es un buen formador.

Ya lo he visto, por lo que debería ir así: Un buen profesor según propone Novoa en 2009, se define como un profesional con conocimiento y dominio del área de desempeño, que posee cultura profesional, que comprende los sentidos de la institución y visión del futuro, tiene tacto pedagógico, comunicación y dimensiones personales para la enseñanza, y que trabaja en equipo: sentido colectivo del trabajo y compromiso social. Ir más allá de la escuela.

Para Jose Manuel Esteve, según comenta en 2009, los docentes docentes se hacen, no nacen y su formación no es un hecho individual, es un hecho colectivo y multidimensional, con una alta responsabilidad de la formación de docentes. Planteó cinco tareas pendientes de las instituciones formadoras: Configurar la identidad profesional, ayudar a comprender el sistema de relaciones, interacciones y comunicación, organizar la enseñanza y adaptar los conocimientos al nivel de los estudiantes para elevarlos a todos hacia el aprendizaje esperado y promover el desarrollo de la capacidad de autoformación.

Como desafíos, los países tienen pendiente realizar políticas de formación docente en el marco de políticas docentes y políticas de remuneraciones que combinen formación, experiencia y responsabilidades.

También asumir con decisión política del más alto nivel las transformaciones estratégicas y sostenidas de la formación docente, formar con calidad a los miles de docentes que se necesitan para una escuela para todos e identificar las mejores estrategias para la formación. Calificó a las universidades como una estrategia del Estado que debe marcar pautas de calidad para la formación de docentes.

Para Mejía «es la hora de comenzar a construir propuestas. Hay que dejar de considerar que mientras no viene la transformación social no son posibles cambios, y que la academia entienda que estos asuntos no se resuelven solo echando discursos». 
LAS REVISTAS EDUCATIVAS PERUANAS Y SU CONTRIBUCIÓN AL ANÁLISIS DE LA PROBLEMÁTICA MAGISTERIAL G. S. PÉREZ POSTIGO, J. L. YANGALI VARGAS Y O. W. TURPO GEBERA

Asistimos a una reconfiguración del saber escolar de la modernidad que ha producido crisis y necesidad de cambiar la educación desde adentro. ¿Cómo se transforma la formación y la evaluación en un mundo que se vuelve conflictivo? Es el gran problema.

Es momento de reflexionar que si dejamos que la educación siga organizada por banqueros, economistas y administradores, nunca va a salir de su condición. Entender que se ha montado una industria del conocimiento, que incluye pruebas estandarizadas como las que promueve el grupo Parsons de los EE. UU., que se están vendiendo al mundo. Ya llegaron a Brasil con una fundación de «buena voluntad».

Esta industria ha generado que haya maestros y maestras sin las acreditaciones suficientes, universidades que convirtieron la formación docente en una mercancía. Así vemos formación de docentes de todo tipo, de calidad de todos los niveles. Todos los temas de moda se vuelven un diplomado, una maestría. Entonces el docente no está siendo objeto de una política formativa.

Estamos en un cambio de época. La crisis también es de las universidades, no solo de los maestros. Aquí hay un problema de fondo. O nos replanteamos el estatuto del docente para hacer una modificación de los procesos de formación y ponemos esa discusión como un problema de la sociedad o seguimos dejándolo en manos de los expertos, continuó Mejía.

Planteó cuatro puntos fundamentales a entender. Con las experiencias internacionales no es posible plantear el proyecto de evaluación o formación si no hay proyecto de país y de educación.

Es necesario construir en el debate la relación con el conocimiento y su importancia en la sociedad del cambio de época. Toca a todo el mundo cambiar los escenarios de lucha sindical, «sacarla de esa mirada estrecha de lo político y gremial que ha tenido en los últimos 40 años. Se tiene que reorganizar para ser un Sindicato de este siglo».

Finalmente, tener bien claro que la pedagogía es el fundamento de la identidad del quehacer del docente. Hoy uno de los lugares centrales de un maestro político es un maestro político-pedagógico. Su práctica lo vuelve sujeto de actuación.

\section{Nuevo milenio, nuevas exigencias editoriales: la indización}

A continuación, damos a conocer los principales medios surgidos en este nuevo siglo cuyo principal rasgo es la búsqueda de su indización. Pretensión que se orienta en lo establecido por el Consejo Nacional de Ciencia, Tecnología e Innovación Tecnológica (Concytec), que clasifica los sistemas de indización en cuatro grupos (20I8). Por orden de importancia, el primer grupo está integrado por las bases de datos scopus y Web of Science (WoS). En el segundo grupo se cuenta a Taylor \& Francis Group, EconLit, ProQuest, Bentham Science. Y en el tercer grupo se encuentran Scielo, DOAJ, Redalyc o Latindex (catálogo). Un cuarto grupo no declarado estaría integrado por las revistas que no se encuentran en ninguna de las bases de datos anteriores. Siguiendo este criterio damos a conocer las revistas educativas peruanas.

RIDU (Revista Digital de Investigación en Docencia Universitaria) es una revista que surge el 2005, una década después que se creara la Universidad Peruana de Ciencias Aplicadas (UPC), universidad que la patrocina. Según el contenido de sus 
LAS REVISTAS EDUCATIVAS PERUANAS Y SU CONTRIBUCIÓN

AL ANÁLISIS DE LA PROBLEMÁTICA MAGISTERIAL

G. S. PÉREZ POSTIGO, J. L. YANGALI VARGAS Y O. W. TURPO GEBERA

primeros artículos resulta evidente su vínculo con temas educativos; en especial con la progresiva presencia de las tecnologías de la información en el sistema educativo global. Los primeros años se privilegian contenidos que orientan la labor del docente universitario, se difunde y apuesta por una administración estratégica; y, en consonancia con lo anterior, se destaca la implementación de tecnologías en los procesos educativos universitarios (Sulmont, 2007; Morgan y Sulmont, 2006; Sulmont, 2005). Vale decirse que, en lo particular, se trata de artículos que dan cuenta de la implementación de un modelo educativo con el que un grupo de universidades privadas emergentes en el contexto neoliberal procuran consolidarse como alternativa educativa; diferenciándose de las universidades privadas instituidas el siglo anterior.

Otro tópico que cabe destacar en las primeras publicaciones de RIDU es su interés por la ética (Narváez, 2007; Vélez, 2006). Como gran parte de las revistas universitarias, su periodicidad no es constante sino hasta el 20I4. Dicho año, implementa las secciones que conserva hasta la fecha (artículos de investigación, artículos de revisión, experiencias docentes, reseñas académicas). Asimismo, a partir del 2014 implementa sus artículos a texto completo en inglés, en muchos de los casos en simultáneo, esto es, en español e inglés. La sección artículos metodológicos se implementará el 2016, año en el que RIDU empieza a formar parte de Emerging Sources Citation Index (ESCI) de Web of Science (WoS). El 2017 fue indizada por Scielo.

RIDU se publica tanto en impreso como en virtual. Sus autores son nacionales (peruanos) y extranjeros. El esfuerzo editorial de la revista se evidencia año tras año. Tiene algunos números temáticos como los que se dedican a la educación inclusiva (2017), la responsabilidad social universitaria (2018), las competencias (2018) y los valores y la ética profesional (2020).

Apuntes Universitarios es una revista educativa de acceso abierto que desde su origen es totalmente en línea. A diferencia de las otras revistas es un medio que solicita a sus autores el pago de una tasa por publicación. Desde su primer número, el 2oiI, muchos de sus artículos ofrecen un enfoque bíblico confesional en general, y educación adventista en lo específico, en consonancia con la universidad que la patrocina (Universidad Peruana Unión) (Gregorutti, 2oI2; Canale, 20II).

$\mathrm{Al}$ iniciar sus actividades la revista tuvo una frecuencia anual, progresivamente fue pasando a ser semestral, cuatrimestral y, finalmente, el 2020, pasó a ser una revista trimestral. En sus primeros números subdividió la revista en secciones: artículos originales, artículos de revisión. Sus últimos números ya no están ubicados en ellas; no obstante, acepta investigaciones empíricas, de revisión, de ensayo, estudios de caso y cartas al editor. Publica artículos en español, portugués e inglés totalmente. Fue la primera revista peruana de contenido educativo en ingresar al Directory of Open Access Journals (DOAJ) el 20I3, actualmente ha sido incorporada al Emerging Sources Citation Index (ESCI) de WoS.

Además de su línea educativa confesional, Apuntes Universitarios también aborda la diversidad de temas que se suscitan en el campo de la investigación educativa, las ciencias sociales y la teología. En el terreno educativo aborda la diversidad temática como la problemática laboral del profesorado, la evaluación, propuestas didácticas y la investigación propiamente dicha.

La Revista Peruana de Investigación Educativa surge el 2009 en formato impreso y virtual. Se trata de una publicación de la Sociedad de Investigación Educativa Peruana (SIEP), por ende, no es una revista vinculada a alguna universidad, sino que forma 
parte de un conjunto de revistas asociadas a organismos no gubernamentales y con influencia en los órganos de gobierno como el Grupo de Análisis para el Desarrollo (GRADE), el Instituto de Estudios Peruanos (IEP) y, en lo educativo, el Consejo Nacional de Educación (CNE). Tuvo una periodicidad anual, no obstante, a partir del 2020 tiene proyectado publicarse dos veces al año. Si bien en sus artículos se abordan diferentes problemáticas educativas, su mayor interés tiene que ver con el desarrollo de políticas. La razón de tal interés tiene que ver con la participación directa de muchos de sus integrantes del equipo editorial o afiliados a la sIEP, en labores de consultoría y de dirección del Ministerio de Educación del Perú.

Los artículos publicados en la revista de SIEP, como más se la conoce, están vinculados a los eventos organizados por dicha institución y de modo especial al Seminario bianual que organiza desde el 2008. Evento que compromete a investigadores nacionales y extranjeros en temas como ruralidad, TIC, desempeño docente, políticas educativas, concepciones y prácticas evaluativas y ciudadanía (Vigo, 2020; Turpo-Gebera, 20II; Turpo-Gebera, 20I2; Alcázar y Valdivia, 20II; Ramírez et al., 2009, entre otros). En razón a su cercanía a la administración del Ministerio de Educación, además del involucramiento de autores extranjeros y cumplimiento de su periodicidad; el 2020 ha sido acogida en el Emerging Sources Citation Index (ESCI).

Las Facultades de Educación del sistema universitario peruano siempre han emprendido la labor editorial. No obstante, su frecuencia siempre ha sido irregular. Lo que ha dado lugar a que muchas de dichas publicaciones no hayan permanecido en el tiempo. Entre las que se han mantenido en la historia se tiene la revista Educación, de la Pontificia Universidad Católica del Perú, la misma que en los últimos años, como las reseñadas en los párrafos precedentes, ha ido realizando un esfuerzo editorial para ser acogida en bases de datos como Scielo (Scientific Electronic Library Online), entre otras. Desde su fundación en I992, Educación de la PUCP se mantuvo en su formato impreso, la adopción del formato digital es reciente, siempre con una periodicidad semestral.

Siguiendo los esquemas internos de tipos de artículo, Educación de la PUCP propuso las secciones de artículos propiamente dichos, comunicaciones y reseñas. La segunda sección fue en su momento innovadora y tenía la misión de dar cuenta de los hallazgos metodológicos y experiencias en el campo educativo (Encinas 1992), en el nuevo siglo se implementó la sección ensayos. En cuanto a sus contenidos publicados la revista Educación se ha caracterizado por su pertinencia contextual. Por ejemplo, en plena violencia política debido a la insurrección armada, la revista abordó su incidencia en la educación (Flores, 1992). Un emblemático número, por el tema abordado en la revista, es el volumen extraordinario de i992, que abordó la problemática de la formación de maestros.

La revista Horizonte de la Ciencia es la continuación de Ciencia y Educación (200720II). Ambas fueron editadas por la Facultad de Educación de la Universidad Nacional del Centro del Perú. Surge el 2ori, vinculada a la Unidad de Posgrado. Adopta el formato virtual desde el 20I5, consiguiendo con ello mayor visibilidad a los artículos. El mismo año decide ampliar sus horizontes y abre su convocatoria a artículos en portugués, además del español. En esta expansión multilingüística a partir del 2017 incluye la traducción en las lenguas locales de la región Junín (quechua, ashaninka y 
LAS REVISTAS EDUCATIVAS PERUANAS Y SU CONTRIBUCIÓN

AL ANÁLISIS DE LA PROBLEMÁTICA MAGISTERIAL

G. S. PÉREZ POSTIGO, J. L. YANGALI VARGAS Y O. W. TURPO GEBERA

nomatsiguenga), del título y palabras clave. Expansión que responde a la asunción de enfoques editoriales incluyentes y decoloniales (Yangali, 2016).

Desde su creación, la periodicidad de Horizonte de la Ciencia sigue siendo la misma: semestral. Las secciones que contiene son Investigación en humanidades, en Ciencias Sociales, en Educación y la de reseñas, siendo el mayor número de sus artículos los referidos a la problemática educativa; procurando ser una revista que integra lenguas, enfoques y disciplinas, también posee una sección de ilustraciones dedicadas a promover a los artistas locales de diferentes áreas como escultura, pintura, cine, teatro, etc.

Su surgimiento acontece en un marco de procura de la acreditación de los programas universitarios (Moya, 20II). Paulatinamente, las revistas y sus artículos han sido indizados en distintas bases de datos, entre las que destacan IRESIE (Índice de Revistas de Educación Superior e Investigación Educativa), ERIHPLus (European Reference Index for the Humanities and Social Sciences) y Redalyc. Entre los temas tratados destacan sus artículos, según el índice generado por Google Académico, sobre pensamiento crítico, procrastinación educativa y gestión educativa.

La revista UCV Hacer es una revista de reciente creación (20I2); forma parte del conjunto de revistas de la Universidad César Vallejo que, al igual que las anteriores (salvo Horizonte de la Ciencia), también es una universidad de administración privada. Como muchas de las revistas de reciente creación, surge en el marco de la aplicación de la Ley 30220, que exige a las universidades cumplir con la misión de fomento de la investigación y no solo la formación de profesionales. Surgió como revista multidisciplinaria de periodicidad semestral; sin embargo, el mayor número de sus artículos tienen que ver con la problemática educativa. En la actualidad es una de las primeras revistas en adoptar la periodicidad continua; no obstante, realiza cortes trimestrales.

UCV Hacer, hasta el 20I7, era una publicación cuyos autores en su mayoría estaban vinculados a la universidad editora. El 2018 implementó la publicación en simultáneo en dos idiomas: inglés y español (Uriarte, 20I8; Robles, 20I8; y otros); sistema que pronto abandonó, limitándose a publicar los artículos en español. El esfuerzo editorial le ha permitido ser indizada por Redalyc.

También de reciente creación y con fines de licenciamiento emergieron las siguientes revistas que a la fecha del presente monográfico han sido acogidas en el Catálogo 2.o de Latindex y dependiendo el esfuerzo de sus editores serán acogidas por otras indizadoras:

I. La revista ConCiencia EPG, de la Escuela de Posgrado de la Universidad de Educación Enrique Guzmán y Valle, que se publica en físico y virtual desde 2016.

2. Eduser, de la Facultad de Educación de la Universidad César Vallejo, que se publica en virtual desde 2014.

3. Hacedor, de la Universidad Señor de Sipán, que se publica en formato virtual desde 2017.

4. Revista Educa UMCH, de la Facultad de Educación y Psicología de la Universidad Marcelino Champagnat, que se publica en virtual desde 2016.

5. Otras revistas de educación que tuvieron dificultades en asuntos de discontinuidad o de migración del formato físico al on-line; y que, sin embargo, resultan relevantes en cuanto a la temática educativa son Educación de la Facultad 
de Ciencias de la Educación de la Universidad Femenina del Sagrado Corazón (UNIFÉ), que se publica en impreso desde 1996 y que a la fecha viene migrando al formato virtual, e Investigación Educativa, de la Facultad de Educación de la Universidad Nacional Mayor de San Marcos, publicada desde 1997 hasta 2013.

\section{Conclusiones}

Para contribuir a resolver la exigente demanda de la formación docente del sindicato docente, a través de la Derrama Magisterial, se impulsó desde 1990 la revista Palabra de Maestro, en cuyas páginas se convocaron especialistas, entre ellos a los dirigentes sindicales, en temas educativos para dar a conocer su visión particular sobre la problemática magisterial como la carrera pública docente, la construcción curricular, la evaluación docente, entre otros temas de interés. Este medio incide, en los contenidos de sus artículos, entrevistas y noticias, en la formación de la opinión crítica del docente frente a las medidas impulsadas por el Gobierno peruano.

Las revistas que emergieron en las últimas décadas se caracterizaron, en sus inicios, por el alto índice de endogamia, es decir, por publicar artículos cuyos autores estaban vinculados con la institución editora o el equipo editorial. No obstante, con la implementación de normas internacionales de indización, en los últimos cinco años, la participación de autores nacionales y extranjeros ajenos a la entidad editora se ha incrementado progresiva y exponencialmente en razón al esfuerzo editorial que tiende a lograr la indización por bases de datos cuya principal exigencia es la internacionalización de la revista.

\section{Bibliografía}

Alcázar, L. y Valdivia, N. (20ir). Descentralización y Presupuesto de la Educación Pública en el Perú: Avances y Desafíos. Revista Peruana de Investigación Educativa, I(3), pp. 7-5I. Recuperado a partir de https://revistas.siep.org.pe/index.php/RPIE/article/view/I5.

Canale, F. (2OII). ¿Epistemología bíblica para la investigación adventista? Una propuesta de trabajo - Biblical Epistemology for Adventist Scholarship? A Working proposal. Apuntes Universitarios, I(I), II9-I4I. https://doi.org/IO.I7162/au.viir.317

CONCYTEC. (2018). Reglamento de calificación, clasificación y registro de los investigadores del sistema nacional de ciencia, tecnología e innovación tecnológica. Recuperado el in de noviembre de 2020, de https://portal.concytec.gob.pe/images/renacyt/reglamento_renacyt_ version_final.pdf.

Encinas RamíreZ, I. (1992). Editorial. Educación, I(I), 5. Recuperado a partir de http://revistas. pucp.edu.pe/index.php/educacion/article/view/4392.

Flores Flores, E. (1992). La escuela en tiempos de guerra. Una mirada a la educación desde la crisis y la violencia, CEAPAZ, TAREA Y IPEDEHP. Educación, $I(2), 232-233$. Recuperado a partir de http://revistas.pucp.edu.pe/index.php/educacion/article/view/4419

Gregorutti, G. (20I2). La Universidad Confesional y los Nuevos Modelos de Universidades: $¿$ Es Posible Mantener la Identidad? - The Confessional University and the New Models of Universities: Is It Possible to Keep Identity? Apuntes Universitarios, 2(I), 9-20. https://doi. org/Io.I7I62/au.voir.304

Morgan Rozas, M. y Sulmont HaAk, L. (2006). Un enfoque estratégico para la gestión del conocimiento en la universidad. La experiencia de la Universidad Peruana de Cien- 
LAS REVISTAS EDUCATIVAS PERUANAS Y SU CONTRIBUCIÓN

AL ANÁLISIS DE LA PROBLEMÁTICA MAGISTERIAL

G. S. PÉREZ POSTIGO, J. L. YANGALI VARGAS Y O. W. TURPO GEBERA

cias Aplicadas (UPC). Revista Digital de Investigación en Docencia Universitaria, 2(I), 23. https://doi.org/Io.19083/ridu.2.26

Moya Rojas, N. (20II). Presentación. Horizonte de la Ciencia, I(I), 3-4. Recuperado a partir de http://revistas.uncp.edu.pe/index.php/horizontedelaciencia/article/view/ı23.

Narváez Rivero, M. (2007). El docente como formador ético. Revista Digital de Investigación en Docencia Universitaria, 3(2), I2. https://doi.org/I0.19083/ridu.3.18

Palabra de Maestro. (2013). Revista Palabra de Maestro, año 22 (59), 9-16.

Palabra de Maestro. (20I4a). Revista Palabra de Maestro, año 23 (6I), II-I4, I6-I9, 22-25.

Palabra de Maestro. (2014b). Revista Palabra de Maestro, año 24 (63), 5-8, I4-17, 21-23.

Palabra de Maestro. (2016). Revista Palabra de Maestro, año 26 (66), I7-I9.

Pérez Postigo, G.; Vilcatoma Salas, L.; Cuadros Paz, L. y Turpo, O. (2020). Los sistemas basados en el pensamiento crítico para el cambio social desde la universidad (pp. I2I-I36). http://www.aisti.eu/Edição/Edition n. ${ }^{\circ}$ E31, 07/2020/ISSN: 1646-9895.

Ramírez, F.; Meyer, J. y Wotipka, C. (2009). Globalización, ciudadanía y educación: Auge y expansión de los marcos de referencia cosmopolitas, multiculturales y de empoderamiento individual. Revista Peruana de Investigación Educativa, I(I), I63-I80. Recuperado a partir de https://revistas.siep.org.pe/index.php/RPIE/article/view/7.

Robles Valdivieso, N. (20I8). Creativity in children of the Pre- Scholar Educational Institution Los Amautas, Sócota. UCV HACER, 7(I). http://revistas.ucv.edu.pe/index.php/ UCV-HACER/article/view/1347/II55

Sulmont HaAk, L. (2005). Recursos educativos digitales: procesos de mediación y mediatización en la comunicación pedagógica. Revista Digital de Investigación en Docencia Universitaria, I(I), I-I9. https://doi.org/IO.I9083/ridu.I.36

Turpo-Gebera, O. (20II). Concepciones y Prácticas evaluativas de los Docentes del Área Curricular de cTA en las II. EE. Públicas de Educación Secundaria de Arequipa (Perú). Revista Peruana de Investigación Educativa, (3), I59-200. https://revistas.siep.org.pe/index.php/ RPIE/article/view/20

Turpo-Gebera, O. (2012). La subjetividad de los docentes de ciencias en la evaluación de los aprendizajes. Revista Peruana de Investigación Educativa, (4), I72-198. https://revistas.siep. org.pe/index.php/RPIE/article/view/30/6I

URIARTE LATORRE, Y. (2018). Three-dimensional psychosocial and-cultural model to develop creativity in primary school. UCV HACER, 7(I). http://revistas.ucv.edu.pe/index.php/ UCV-HACER/article/view/I336/II42

Vélez VAlcárceL, O. (2006). La relación profesor-alumno en el aula como espacio de formación ética en la universidad. Revista Digital de Investigación en Docencia Universitaria, 2(I), 2I. https://doi.org/IO.I9083/ridu.2.25

Vigo, B. N. (2020). Efecto del aumento de la oferta educativa en la escolaridad y el trabajo adolescente en el Perú rural. Revista Peruana de Investigación Educativa, I(I2), 55-88. Recuperado a partir de https://revistas.siep.org.pe/index.php/RPIE/article/view/I29

Yangali Vargas, J. L. (20I6). Presentación [La educación hacia el bicentenario: inclusión]. Horizonte de la Ciencia, 6(II), 7-I2. Recuperado a partir de http://revistas.uncp.edu.pe/index.php/horizontedelaciencia/article/view/30I 
